\title{
Eccema de labios. Experiencia en la Unidad de Eccema de Contacto de un centro terciario español
}

\section{Lip eczema. Experience in the Contact Dermatitis Unit of a Spanish tertiary hospital}

\author{
L. Loidi-Pascual, M. Hervella-Garcés, M. Larrea-García, R. Santesteban-Muruzábal, \\ A. Agulló-Pérez, I. Yanguas-Bayona
}

\section{RESUMEN}

Fundamento. El eccema labial es un problema poco frecuente en las Unidades de Eccema de Contacto (UEC). Hasta el momento han sido publicadas escasas series que muestren el perfil de estos pacientes y las causas de su eccema, ninguna de ellas española. El objetivo de este estudio fue el de analizar el perfil epidemiológico de los pacientes que acuden a la UEC en nuestro entorno, los diagnósticos principales y los alérgenos relevantes.

Material y métodos. Se realizó una revisión retrospectiva de los pacientes remitidos a la UEC en el periodo 2005-2014. Se realizaron pruebas epicutáneas con la serie estándar ampliada del Grupo Español de Investigación en Dermatitis Alérgica de contacto (GEIDAC), productos propios y otras baterías complementarias.

Resultados. En los 78 pacientes estudiados, los diagnósticos más frecuentes fueron la dermatitis de contacto alérgica y la dermatitis atópica. Los alérgenos relevantes más frecuentes fueron los cosméticos y los fármacos tópicos.

Conclusiones. Es fundamental estudiar a los pacientes con eccema labial con pruebas epicutáneas para poder filiar correctamente su etiología y según esto, realizar un tratamiento más adecuado.

Palabras clave. Dermatitis alérgica de contacto. Eccema. Pruebas epicutáneas. Cosméticos.

\begin{abstract}
Background. Lip eczema is an infrequent problem in Contact Dermatitis Units (CDU). Very few series have been published to date that show the profiles of such patients and the causes of their eczemas, and none are Spanish. The goal of this study was to analyze the epidemiological profile of the patients who attend a CDU in our setting, the main diagnoses and the relevant allergens.
\end{abstract}

Methods. A retrospective review was made of the patients referred to the CDU in the 2005-2014 period Patch tests were carried out with the extended standard series of the Spanish Research Group on Allergic Contact Dermatitis (Grupo Español de Investigación en Dermatitis Alérgica de contacto - GEIDAC), our own products and other complementary sets.

Results. The most frequent diagnoses in the 78 patients studied were allergic contact dermatitis and atopic dermatitis. The most frequent relevant allergens were cosmetics and topical medications.

Conclusions. It is essential to study the patients with lip eczema with patch tests to be able to correctly determine their etiology and accordingly to carry out the most suitable treatment.

Keywords. Cheilitis. Allergic contact dermatitis. Eczema. Patch tests. Cosmetics.
Servicio de Dermatología. Complejo Hospitalario de Navarra. Pamplona.

Recepción: 18 de diciembre de 2014

Aceptación provisional: 15 de abril de 2015

Aceptación definitiva: 7 de septiembre de 2015

\section{Correspondencia:}

Leire Loidi Pascual

Servicio de Dermatología

Complejo Hospitalario de Navarra

Irunlarrea, 3

31008 Pamplona

E-mail: leyre.loidi.pascual@cfnavarra.es 


\section{INTRODUCCIÓN}

El eccema labial puede deberse a múltiples causas, tanto endógenas como exógenas. Hasta el momento han sido publicadas escasas series de pacientes con este cuadro clínico ${ }^{1-9}$, siendo las que aportan un mayor número de pacientes las provenientes de Singapur ${ }^{1,4}$, Reino Unido ${ }^{5}$ y Estados Unidos $^{8}$. De estos datos se concluye que en general, el eccema labial es un motivo de consulta poco frecuente en las Unidades de Eccema de Contacto (UEC), con una prevalencia del 1 al 3,4\%. Entre los pacientes estudiados, la mayoría son mujeres de edad media y $30-46 \%$ tenían diagnóstico previo de atopia ${ }^{1-9}$.

Las principales causas de eccema labial son la dermatitis atópica y la dermatitis alérgica de contacto. Los alergenos relevantes más frecuentes son, entre otros, los dentífricos, los perfumes (Fragance Mix I y bálsamo de Perú), la colofonia y el níquel ${ }^{1-9}$.

Los principales objetivos de este trabajo son analizar el perfil epidemiológico de los pacientes que acuden a la UEC, los diagnósticos principales tras el estudio y los alérgenos relevantes.

Tras realizar una búsqueda bibliográfica exhaustiva en las principales bases de datos especializadas, no hemos encontrado más estudios que muestren series de similares características procedentes de un hospital español.

\section{MATERIAL Y MÉTODOS}

Se realizó una revisión retrospectiva de los pacientes remitidos para estudio de queilitis a la UEC del Servicio de Dermatología y que padecieran eccema labial de manera aislada o asociado a eccema en otras localizaciones. Todos ellos fueron derivados a esta Unidad por especialistas en Dermatología. En el periodo de 2005 a 2014 se estudiaron 78 pacientes y a todos se les realizó una completa anamnesis, exploración física y analítica sanguínea en el caso de que fuera necesario (IgE en caso de sospecha de dermatitis atópica). Así mismo, a todos ellos se les parcheó la serie estándar ampliada del Grupo Español de Investigación en Derma- titis Alérgica de Contacto (GEIDAC) ${ }^{10}$, los productos propios en caso de disponer de ellos y otras baterías complementarias según la sospecha clínica (sobre todo la serie de cosméticos), suministradas por los laboratorios Chemotechnique Diagnostics $\mathrm{AB}$ y J. Martí Tor (Barcelona). La lectura de las pruebas se realizó el D2, D4 y el D7 de forma opcional. La lectura de las mismas se realizó según la escala de la International Contact Dermatitis Research Group (ICDRG). El diagnóstico de dermatitis atópica se llevó a cabo de acuerdo con los criterios diagnósticos de consenso de la Academia Americana de Dermatología de $2003^{11}$. La relevancia de la positividad de las pruebas epicutáneas se establece según la presencia del alérgeno en productos que se haya aplicado previamente el paciente y según la mejoría clínica que presente tras la retirada del mismo.

En cuanto al análisis comparativo de los datos, en los casos en los que se contrastaron diferentes características entre grupos, se realizó una Chi cuadrado para la comparación de proporciones y en el caso de que no se cumplieran los requisitos necesarios, el test exacto de Fisher.

\section{RESULTADOS}

Los pacientes estudiados por queilitis en esta unidad fueron el $6 \%$ del total de parcheados en este periodo. El 87,18\% eran mujeres, con un rango de edad de 7 a 86 años (media 41,64). El tiempo de evolución medio de las lesiones era de 25 meses (mediana de 11). El índice MOAHLFA (Male, Occupational, Atopy, Hand, Leg, Face, Age >40) de los pacientes se muestra en la tabla 1 . Cabe destacar la diferencia en el porcentaje de mujeres $(87,18 \%)$ y antecedentes de dermatitis atópica $(28,21 \%)$ comparando con el total de pacientes estudiados durante este periodo.

Los diagnósticos principales más frecuentes tras el estudio completo fueron la dermatitis atópica (DA) (30\%) y la de contacto alérgica (DCA) (27\%), seguido de la irritativa (DCI) (13\%) (Fig. 1). Los demás diagnósticos abarcaban la dermatitis perioral, otros eccemas como la dermatitis seborreica y la candidiasis oral crónica. 
Tabla 1. Índice MOAHLFA. Comparativa de los pacientes con queilitis frente al total de los estudiados

\begin{tabular}{lcc}
\hline & Cheilitis\% (IC 95\%) & Total estudiados\% (IC 95\%) \\
\hline Male & $12,82(5,4-20,2)$ & $39,2(36,5-41,9)$ \\
\hline Occupational & $5,13(0,2-9,9)$ & $9,2(7,6-10,8)$ \\
\hline Atopy & $28,21(18.2-38.2)$ & $16,7(14,6-18,7)$ \\
\hline Hand & $17,95(12,5-20,9)$ & $41,7(39,1-44,5)$ \\
\hline Leg & $1,28(0-3,8)$ & $11,6(9,3-12,8)$ \\
\hline Face & $100(96,2-100)$ & $28,3(25,8-30,8)$ \\
\hline Age $>40$ & $52,56(38,9-61,1)$ & $60,9(58,2-63,6)$ \\
\hline Total & 78 & 1.266 \\
\hline
\end{tabular}

MOAHLFA: acrónimo utilizado para describir las características de la población estudiada.

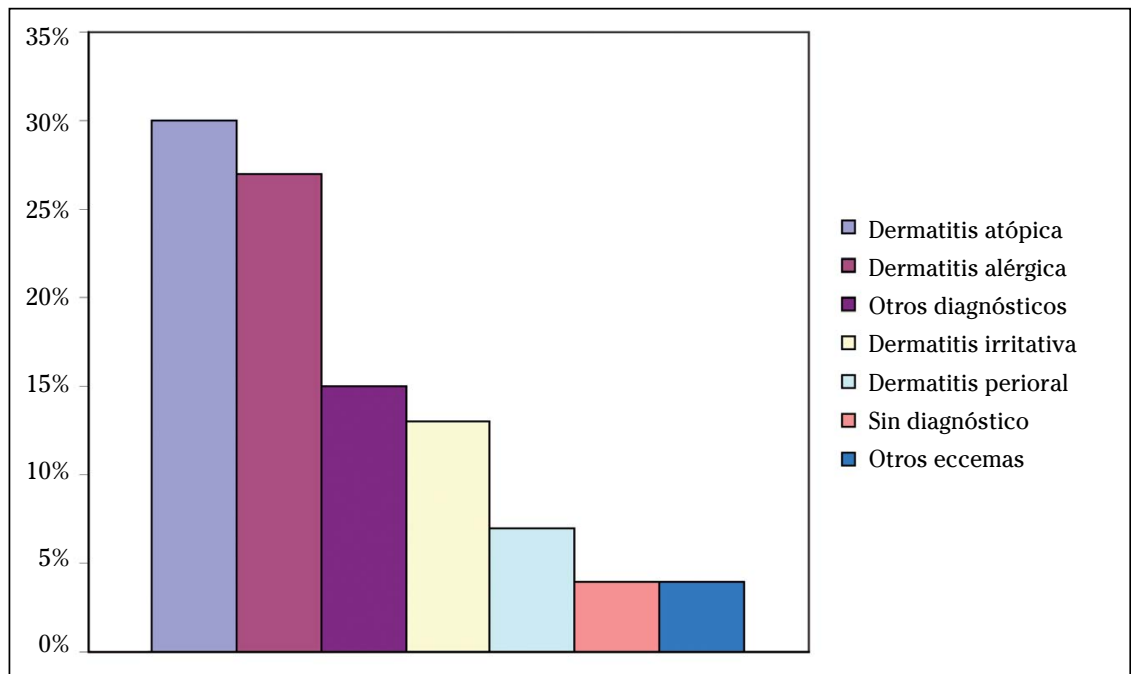

Figura 1. Diagnósticos principales de los pacientes estudiados.

Al separar a los pacientes según las edades, se observó que en pacientes menores de 30 años la causa más frecuente de queilitis es la atopia. Sin embargo, en mayores de 30 años los diagnósticos se encontraban más repartidos, siendo los más frecuentes la dermatitis atópica y la alérgica de contacto. Estas diferencias entre estos grupos de edad fueron estadísticamente significativas ( $\mathrm{p}<0,001)$ (Fig. 2).

En cuanto a la queilitis alérgica de contacto, 54 pacientes mostraron al menos una prueba positiva en las pruebas epicutáneas, de las cuales 29 fueron relevantes. En la figura 2 se detallan los grupos de alérgenos relevantes. Los más frecuentes fueron los cosméticos (38\%), las plantas (21\%), los fármacos (18\%), y los perfumes (14\%).

En la tabla 2 se muestran los alérgenos relevantes detallados de forma individual. Los alérgenos más específicos de la localización labial fueron los galatos -OR 29,23 (IC 95\% 8,36/102,19)-, las plantas compuestas -OR 5,91 (1,5-22,7)- y los antivirales tópicos -OR 11,86 (2,61-53,96)-.

La batería estándar tan solo detectó el $36 \%$ de los alérgenos relevantes, por lo que fueron necesarias la estándar ampliada (14\%), otras adicionales (29\%, sobre todo cosméticos) y los productos propios (21\%) para detectar el resto de alérgenos. 


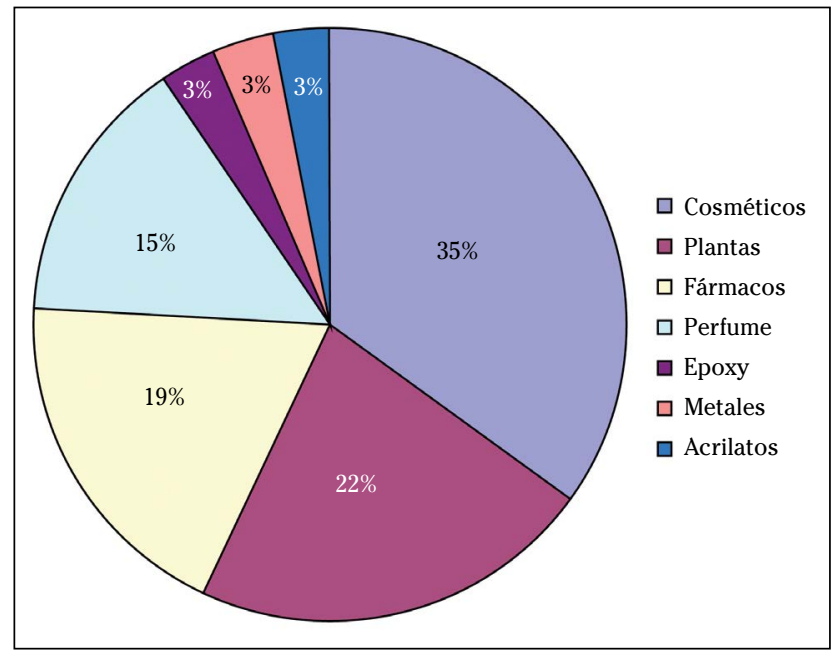

Figura 2. Alérgenos relevantes.

Tabla 2. Listado de alérgenos relevantes vistos de manera individual

\begin{tabular}{|c|c|c|c|}
\hline & & Alérgeno & $\mathrm{N}^{o}$ de casos relevantes \\
\hline \multirow[t]{7}{*}{ Cosméticos } & \multirow{3}{*}{$\begin{array}{l}\text { Conservantes- } \\
\text { antioxidantes }\end{array}$} & Galatos & 4 \\
\hline & & Formol & 1 \\
\hline & & MCI & 1 \\
\hline & \multirow[t]{2}{*}{ Vehículos } & Lanolina & 2 \\
\hline & & DMAPA & 1 \\
\hline & \multirow[t]{2}{*}{ Otros } & Persulfato amónico & 1 \\
\hline & & $\mathrm{SSO}$ & 1 \\
\hline \multirow[t]{3}{*}{ Plantas } & & Plantas compuestas & 3 \\
\hline & & Líquenes & 2 \\
\hline & & Madera cocobolo & 1 \\
\hline \multirow[t]{4}{*}{ Fármacos } & & Tromantadina & 2 \\
\hline & & Peróxido de benzoilo & 1 \\
\hline & & Captopril & 1 \\
\hline & & Aciclovir & 1 \\
\hline \multirow[t]{4}{*}{ Perfumes } & & Lyral & 1 \\
\hline & & Fragance Mix I & 1 \\
\hline & & Geraniol & 1 \\
\hline & & Oakmoss & 1 \\
\hline Metales & & Niquel & 1 \\
\hline Epoxy & & Epoxy & 1 \\
\hline Acrilatos & & Laca de uñas propia & 1 \\
\hline
\end{tabular}

MCI: metilcloroisotiazolinona SSO: sorbitan sesquioleato DMAPA: dimetilaminopropilamina 


\section{DISCUSIÓN}

En general, el eccema labial es un motivo de consulta poco frecuente en las unidades de eccema de contacto. Sin embargo, en nuestro estudio hemos encontrado una prevalencia algo mayor (6\%) a las series previamente publicadas, que mostraban una frecuencia del $1-3,4 \%^{1-9}$. En esta serie los datos demográficos de la po- blación estudiada fueron similares, observándose una alta frecuencia de mujeres de edad media y una prevalencia de dermatitis atópica en torno al $37 \%$. En la tabla 3 se muestra un resumen de las series publicadas hasta el momento de pacientes con queilitis.

En nuestra UEC la distribución de los diagnósticos más frecuentes fueron la DA, la DCA y la DCI.

Tabla 3. Resumen de las series previamente publicadas.

\begin{tabular}{|c|c|c|c|c|}
\hline $\begin{array}{c}\text { Serie } \\
\text { (localización } \\
\text { y año) }\end{array}$ & $\begin{array}{c}n \\
(\% \text { del } \\
\text { total) }\end{array}$ & $\begin{array}{c}\text { Datos } \\
\text { demográficos }\end{array}$ & Diagnósticos & Alergenos relevantes \\
\hline $\begin{array}{l}{ }^{1} \text { Singapur } \\
\text { (1992) }\end{array}$ & 27 & $\begin{array}{l}\text { Mujeres } 78 \% \\
40,7 \% 20-29 \text { años } \\
\text { Atopia } 33,3 \%\end{array}$ & $\begin{array}{l}\text { DCA } 33 \% \\
\text { Endógena } 33 \% \\
\text { Desconocida } 33 \%\end{array}$ & $\begin{array}{l}\text { Dentífricos } \\
\text { Barras labiales (lanolina, ácido ricinoleico, } \\
\text { níquel) }\end{array}$ \\
\hline $\begin{array}{l}{ }^{2} \text { Australia } \\
(1999)\end{array}$ & $75(3,4 \%)$ & $\begin{array}{l}\text { Mujeres } 71 \% \\
\text { Edad mediana } 41 \\
\text { años }\end{array}$ & $\begin{array}{l}\text { DCI } 36 \% \\
\text { DCA } 25 \% \\
\text { DA } 19 \% \\
\text { Eccema de causa } \\
\text { desconocida } 9 \% \\
\text { Otros } 9 \% \\
\text { DS } 1 \% \\
\end{array}$ & $\begin{array}{l}\text { - Fármacos tópicos } \\
\text { - Barras labiales (isoeugenol, Fragance Mix) } \\
\text { - Fotoprotectores (oxibenzona) } \\
\text { - Dentífricos (triclosan, menta) } \\
\text { - Colofonia } \\
\text { - Barnices de uñas } \\
\text { - Instrumento musical (níquel) }\end{array}$ \\
\hline${ }^{3}$ India (1999) & 8 & $\begin{array}{l}\text { Mujeres } 75 \% \\
\text { Atopia } 38 \% \\
\text { Edad media } 25 \text { años }\end{array}$ & DCA $75 \%$ & $\begin{array}{l}\text { (relevancia no mencionada) } \\
\cdot \text { Galatos } \\
\cdot \text { Tiomersal } \\
\cdot \text { Mercurio } \\
\text {. Sorbitan monooleato }\end{array}$ \\
\hline $\begin{array}{l}{ }^{4} \text { Singapur } \\
(2000)\end{array}$ & 202 & $\begin{array}{l}\text { Mujeres } 90 \% \\
\text { Edad media } 31 \text { años }\end{array}$ & $\begin{array}{l}\text { Endógena } 53 \% \\
\text { DCA } 34 \% \\
\text { DCI } 5.4 \%\end{array}$ & $\begin{array}{l}\text { Cosméticos labios (ac. ricinoleico) } \\
\text {. Dentífricos } \\
\text {. Fragance Mix }\end{array}$ \\
\hline $\begin{array}{l}{ }^{5} \text { Reino Unido } \\
\text { (2003) }\end{array}$ & $146(1,5 \%)$ & $\begin{array}{l}\text { DCA: mujeres } 95 \% \text {, } \\
\text { edad media } 44 \text { años, } \\
\text { atopia } 45 \%\end{array}$ & DCA $22 \%$ & $\begin{array}{l}\text { Fragance Mix } \\
\text { Shellac } \\
\text {. Colofonia } \\
\text {. M. Pereirae }\end{array}$ \\
\hline${ }^{6}$ Italia (2006) & 83 & $\begin{array}{l}\text { Mujeres } 71 \% \\
\text { Edad media } 40 \text { años } \\
\text { Atopia } 35 \%\end{array}$ & $\begin{array}{l}\text { DCI } 37 \% \\
\text { DA } 26 \% \\
\text { DCA } 18 \%\end{array}$ & $\begin{array}{l}\text { Fragance Mix } \\
\text { - Niquel } \\
\text { - Euxyl K400 } \\
\text { - M. Pereirae } \\
\text { - Cosméticos propios }\end{array}$ \\
\hline${ }^{7}$ Italia (2008) & 129 & $\begin{array}{l}\text { Mujeres } 82 \% \\
\text { Edad media } 42 \text { años }\end{array}$ & $\begin{array}{l}\text { DCA } 65 \% \\
\text { DA } 19 \% \\
\text { DCI } 16 \%\end{array}$ & $\begin{array}{l}\text {. Niquel } \\
\text { Fragance Mix } \\
\text { M. Pereirae } \\
\text {. Neomicina }\end{array}$ \\
\hline${ }^{8}$ EEUU (2008) & $196(2 \%)$ & $\begin{array}{l}\text { Mujeres } 84 \% \\
\text { Edad media } 47 \text { años } \\
\text { Atopia } 37 \%\end{array}$ & $\begin{array}{l}\text { DCA } 38 \% \\
\text { DCI }\end{array}$ & $\begin{array}{l}\text {. Fragance Mix } \\
\text {. Niquel }\end{array}$ \\
\hline $\begin{array}{l}{ }^{9} \mathrm{Grecia} \\
(2008)\end{array}$ & $106(1 \%)$ & $\begin{array}{l}\text { Mujeres } 75,5 \% \\
\text { Edad media } 35 \text { años } \\
\text { Atopia } 46 \%\end{array}$ & DCA & $\begin{array}{l}\text { - Niquel } \\
\text { - M. Pereirae } \\
\text { - Colofonia } \\
\text { - Formaldehído } \\
\text { - Galatos }\end{array}$ \\
\hline
\end{tabular}

DCI: dermatitis de contacto irritativa; DCA: dermatitis de contacto alérgica; DA: dermatitis atópica; DS: dermatitis seborreica 


\section{Galatos}

De forma similar a otras series, los alérgenos relevantes más frecuentes fueron los cosméticos. Sin embargo, es llamativa la falta de similitud entre los alérgenos de forma individual. Las fragancias fueron un alérgeno relevante en cuatro casos (14\%) y el níquel en tan solo uno ( $3 \%$ de todas las queilitis alérgicas de contacto). Ningún caso se debió a alergia al bálsamo de Perú o la colofonia.

Dentro de los cosméticos, cabe mencionar a los galatos, que fueron el alérgeno más frecuente en nuestro caso (36\% de todos los cosméticos relevantes). Estos son sustancias antioxidantes que se utilizan como componentes de productos cosméticos, fármacos tópicos y alimentos. Son ésteres alquílicos del ácido gálico y se diferencian entre sí por su cadena lateral. Su función es evitar la oxidación de los ácidos grasos insaturados que contienen. Los principales galatos empleados en la industria son el galato de propilo, el galato de octilo y el galato de dodecilo. A pesar de su ubicuidad tanto en productos tópicos como en alimentos, el número de casos de dermatitis alérgica de contacto por galatos es relativamente escaso ${ }^{12,13}$. En teoría, el incremento en la longitud de su cadena lateral se correlaciona con el aumento de su capacidad de sensibilizar, siendo el dodecil galato el que presenta mayor capacidad sensibilizante ${ }^{14}$. En la práctica clínica parece que el mayor porcentaje de dermatitis alérgica de contacto se debe al propil galato. Esto puede deberse a que es el más utilizado en la industria. En cuanto a los casos de DCA por galatos en España, cabe destacar la serie de García-Melgares y coll $^{15}$, donde recogieron 46 casos de este cuadro clínico.

En nuestra serie, todos los casos se dieron en mujeres de 45-50 años sin antecedentes de dermatitis atópica. Los alérgenos fueron relevantes definitivos o probables y el galato responsable se encontraba en la barra labial que utilizaba previamente la paciente. Ningún caso se consideró ocupacional.
Aunque en nuestro estudio éste fue un alérgeno muy importante, previamente tan solo se nombra en dos series realizadas en India $^{3}$ y Grecia ${ }^{9}$.

\section{Plantas}

Las plantas son un componente ampliamente utilizado en los productos cosméti$\cos ^{16}$. Entre éstas, una de las familias más importantes es la de las plantas compuestas o Compositae. De entre sus componentes, las sustancias con mayor capacidad sensibilizante son las sesquiterpenolactonas. Aunque estas plantas son ampliamente utilizadas en productos cosméticos y fármacos tópicos, lo más típico es que produzca una dermatitis de predominio facial aerotransportada en trabajadores al aire libre (agricultores, granjeros, jardineros...).

Del total de pacientes con queilitis alérgica de contacto, en nuestra serie se observó un $21 \%$ de pacientes alérgicos a las plantas. Entre éstos, el 50\% eran alérgicos a las plantas compuestas ( 3 casos), el 33\% a los líquenes ( 2 casos) y el $17 \%$ a la madera (1 caso).

\section{Antivirales tópicos}

Teniendo en cuenta la amplia utilización de los antivirales tópicos, el número de DCA debidos a estos fármacos publicados es baja ${ }^{17}$. La tromantadina es un potente sensibilizante $(40 \%$ de los casos con queilitis alérgica de contacto a fármacos de nuestra serie), con un amplio número de casos de alergia de contacto descritos hasta el momento ${ }^{17}$. Sin embargo, a pesar de que el aciclovir se usa más frecuentemente, es un sensibilizante menos potente (20\% en nuestra serie). Una DCA a un preparado de aciclovir tópico, puede deberse a alergia al propio fármaco o algún componente del preparado, como el propilenglicol. Los pacientes alérgicos a este fármaco pueden desarrollar reacciones sistémicas con el uso por vía oral de aciclovir, valaciclovir, ganciclovir y famciclovir, por lo que la única alternativa válida sería el foscarnet o el cidofovir ${ }^{18}$. 
Las diferencias en los alérgenos pueden deberse por un lado a que las series publicadas previamente proceden de estudios realizados en los años 1992 a 2008, con lo que los componentes de los cosméticos y los hábitos de utilización de éstos podrían diferir respecto a los actuales, sobre todo los de las primeras series. Por otro lado, esta desigualdad entre los álergenos también puede deberse a las diferencias en los hábitos entre las poblaciones estudiadas y la nuestra.

\section{Barras labiales}

La principal fuente de dermatitis de contacto en nuestra serie fueron los productos cosméticos labiales. Estos son ampliamente utilizados, tanto en forma de perfiladores, barras o hidratantes labiales. Según una revisión realizada por Scheman y col$^{19}$, los alérgenos más frecuentemente contenidos en estos productos son los derivados de la vitamina $\mathrm{E}$, los parabenos, el propóleo, el ácido sórbico, el sorbitan sesquioleato (SSO), los perfumes y la lanolina. Dado el amplio uso de éstos, no es raro que en ocasiones den problemas de eccema de contacto. En nuestro estudio observamos cuatro casos de queilitis alérgicas de contacto debidas a perfumes (14\%), dos a lanolina $(6,8 \%)$ y uno a SSO $(3,4 \%)$.

Hay que tener en cuenta que este estudio cuenta con un número limitado de pacientes, por lo que para poder tener conclusiones más firmes sería necesario tener más datos de otros centros. Sin embargo, pensamos que puede ser de utilidad para dermatólogos que vean pacientes con similares características a los descritos previamente, en áreas geográficas cercanas, para la orientación diagnóstica de los pacientes con eccema labial.

Ante un paciente con una dermatitis labial es fundamental realizar en primer lugar una correcta anamnesis y exploración cutánea, para poder excluir otras posibles causas de queilitis, tales como la queilitis actínica, infecciosa, granulomatosa, glandular, apostematosa... Es difícil establecer el diagnóstico de una queilitis solo con las características clínicas. Por ello, una vez realizada la sospecha de eccema, será fundamental la realización de pruebas epicutáneas para concretar el diagnóstico. Esto es importante ya que dependiendo de la causa subyacente, el manejo terapéutico de la queilitis será diferente. En nuestra serie, con la batería estándar tan solo se encontró el alérgeno responsable de la DCA en un $36 \%$ de los casos. Por ello, además de ésta, es fundamental parchear los productos propios sospechosos y series adicionales como los cosméticos para llegar a identificar el alérgeno causante del cuadro clínico.

\section{BIBLIOGRAFÍA}

1. Lim JTE, NG SK, GoH CL. Contact cheilitis in Singapore. Contact Dermatitis 1992; 27: 263264.

2. Freeman S, Stephens R. Cheilitis: analysis of 75 cases referred to a contact dermatitis clinic. Am J Contact Dermat 1999; 10: 198-200.

3. Kanthraj GR, Shenol SD, SRinivas CR. Patch testing in contact cheilitis. Contact Dermatitis. 1999; 40: 285.

4. Lim SH, GoH CL. Epidemiology of eczematous cheilitis at a tertiary dermatological referral centre in Singapore. Contact Dermatitis 2000; 43: 322-326.

5. Strauss RM, Orton DI. Allergic contact cheilitis in the United Kingdom: a retrospective study. Am J Contact Dermat 2003; 14: 75-77.

6. Zoli V, Silvani S, Vincenzi C, Tosti A. Allergic contact cheilitis. Contact dermatitis. 2006; 54: 296-297.

7. Schena D, Fantuzzi F, Giromoloni G. Contact allergy in chronic eczematous lip dermatitis. Eur J Dermatol 2008; 18: 688-692.

8. Zug Ka, Kornik R, Belsito Dv, Deleo VA, Fowler JR. JF, MAIBACH HI et al. Patch- testing North American lip dermatitis patients: data from the North American Contact Dermatitis Group, 2001 to 2004. Dermatitis 2008; 19: 202208.

9. Katsarou A, Armenaka M, Vosynioti V, Lagogiann E, Stavropoulos PG, Kalogeromitros D. Allergic contact dermatitis in Athens. Contact Dermatitis 2008; 59: 123-125.

10. Hervella M, Fernández-Redondo V. La dermatitis de contacto en el siglo XXI. La apuesta del Grupo Español de Investigación en Dermatitis de Contacto y Alergia Cutánea (GEIDAC). Actas Dermosifiliogr 2012;103: 345-347. 
11. Eichenfield LF, Hanifin JM, Luger TA, Stevens SR, PRIDE HB. Consensus conference on pediatric atopic dermatitis. J Am Acad Dermatol 2003; 49: 1088-1095.

12. Muñoz D, Audicana M, Gastaminza G, Fernández E. Dermatitis de contacto por galatos. Alergol Inmunol Clin 2002; 17: 173-177.

13. Schnuch A, Geier J, Uter W, Frosch PJ. Patch testing with preservatives, antimicrobials and industrial biocides. Results from a multicentre study. Br J Dermatol 1998; 138: 467476.

14. Hausen BM, Beyer W. The sensitiziting capacity of the antioxidants propyl, octyl, and dodecyl gallate and some related gallic acid esters. Contact Dermatitis 1992; 26: 253-258.

15. García-Melgares ML, De la Cuadra J, Martin B, Laguna C, Martinez L, Alegre V. Sensibilización por galatos. Revisión de 46 casos. Actas dermosifiliogr 2007; 98: 688-693.

16. JACK AR, Norris PL, STORRs FJ. Allergic contact dermatitis to plant extracts in cosmetics. Semin Cutan Med Surg 2013; 32: 140-146.

17. Holdiness MR. Contact dermatitis from topical antiviral drugs. Contact dermatitis 2001; 44: 265-269.

18. Vernassiere C, Barbaud A, Trechot PH, WeberMuller F, Schmutz JL. Systemic acyclovir reaction subsequent to acyclovir contact allergy: which systemic antiviral drug should then be used? Contact Dermatitis 2003; 49: 155157.

19. Scheman A, Jacob S, Katta R, Nedorost S, WarSHAW E, ZiRWAS M et al. Lip and common dental care products: trends and alternatives. Data from the American contact alternatives group. J Clin Aesthet Dermatol 2011; 4: 50-53. 